BMJ Open Sport \& Exercise Medicine

\section{Inferior heel pain in soccer players: a retrospective study with a proposal for guidelines of treatment}

To cite: Saggini R, Migliorini M, Carmignano SM, et al. Inferior heel pain in soccer players: a retrospective study with a proposal for guidelines of treatment. BMJ Open Sport \& Exercise Medicine 2018;4:e000085. doi:10.1136/ bmjsem-2015-000085

Accepted 3 July 2016
Check for updates

${ }^{1}$ Department of Medical Oral and Biotechnological Sciences, 'G d'Annunzio' University, Chieti, Italy

${ }^{2}$ Dipartimento di Medical Orale and Biotechnological Sciences, University G. d'Annunzio of Chieti-Pescara, Chieti, Italy ${ }^{3}$ School of Specialty in PRM, 'G d'Annunzio' University, Chieti, Italy

${ }^{4}$ Department of Medicine and Science of Aging, 'G d'Annunzio' University, Chieti, Italy

Correspondence to Prof Raoul Saggini; saggini@ unich.it

\section{ABSTRACT}

Background The cause of heel pain among soccer players is multifactorial and is related to repetitive microtrauma due to impact forces involving technical moves, but also the playground, the exercise mode, the recovery time, the climatic conditions and the footwear used.

Aim To investigate the aetiology of plantar heel pain of soccer players with the objective of proposing an example of guidelines for treatment.

Methods We investigated the prevalence and characteristics of inferior heel pain of 1473 professional, semiprofessional and amateur players. All evaluated subjects were submitted to a specific rehabilitation protocol that involved advanced physical therapies and viscoelastic insoles depending on the aetiology of pain.

Results Clinical and instrumental examinations revealed that 960 of 1473 athletes had inferior heel pain. These patients were divided into seven groups based on aetiology: sural nerve compression, abductor digiti minimi compression, atrophy and inflammation of the fat pad, plantar fasciitis, stress injury of the heel spur, stress fracture of the heel bone and heel spur. The proposed rehabilitation treatment aims for a reduction of pain and an early return to sports, with excellent results.

Conclusions According to what was observed in the present study, related also to the specific treatment of inferior heel pain, and considering the technological progress achieved in recent years, we can now propose an integrated therapeutic approach to treatment of heel pain, properly differentiated according to specific aetiology.

\section{INTRODUCTION}

Soccer, one of the most popular team sports in the world with over 200 million registered players worldwide, is a game of non-stop action. Most injuries in soccer occur in the lower limb, mostly in the knees, feet and ankles. Repeated or prolonged use (overuse) injuries are common problems. These injuries are the result of constant overloading and wearing down of a tendon, muscle, bone or joint, resulting in the inability of that structure to perform its normal biomechanical functions.

Previous studies demonstrated a high injury rate associated with soccer. Suzue et $a t^{2}$

\section{What are the new findings?}

The retrospective nature of the study may constitute a limitation.

- Treatment strategies and recommendations should be specific to any heel pain etiology.

- Knowledge of all clinical conditions related to inferior heel pain will allow an accurate diagnosis and reduce the costs of inappropriate diagnostic investigations.

- Specific treatments using advanced physical energies can result in shorter recovery times with improved quality of life for patients and a faster return to sports among athletes.

in a study about prevalence of soccer-related overuse injuries revealed that a large number of players had heel and anterior knee pain, yet only $26.9 \%$ of them chose to undergo an imaging examination.

The risk factors for soccer-related injuries include age, gender, skill level, environment and surface type.

The cause of injury is multifactorial and is related to repetitive microtrauma due to impact forces involving technical moves, but also the playground, the exercise mode, the recovery time, the climatic conditions and the footwear used. ${ }^{3}$

The most common technical moves are tackling and being tackled, sprinting, running, twisting and turning, jumping, landing, and kicking the ball. They are all complex movement patterns that require a high grade of coordination and body control.

In a review, Laurie et at showed that comparison of different studies is difficult because of the different classification systems used to indicate severity of injury; some studies classified on the basis of the medical treatment used, while others on the days of absence from the competition. ${ }^{5}$

Based on the latest guidelines in 2010 developed by the clinical practice guidelines Heel Pain Committee of the American College of Foot and Ankle Surgeons (ACFAS), heel pain 
is divided into two main groups: plantar or inferior, and posterior heel pain. ${ }^{6}$

Plantar or inferior heel pain causes soreness or tenderness at the sole of the foot under the heel, which sometimes extends into the medial arch. ${ }^{7}$ This condition has been known for many years as 'plantar fasciitis', but this name was abandoned about 10 years ago because research indicated that it was not an inflammatory condition. Hence, the term 'plantar fasciosis' was suggested, which means 'degeneration' of the plantar fascia. Since then, some authors have also referred to it as plantar fasciopathy, which generically indicates 'a pathology of the plantar fascia'. ${ }^{8}$ However, recent imaging studies have increasingly demonstrated that the condition affects more structures than just the plantar fascia (eg, the heel bone, surrounding tissues, nerve), so the general term 'inferior heel pain' seems to be more appropriate.

The classification of heel pain should be of an etiological type so that the clinician can make a specific recommendation in relation to the cause that determined it.

In our study, we investigated the aetiology of plantar heel pain among soccer players, with the aim of proposing an example of guidelines for treatment. We carried out a retrospective epidemiological investigation on soccer players with pain on inferior heel surface who came for observation between 1992 and 2012.

\section{MATERIALS AND METHODS}

We investigated the prevalence and characteristics of inferior heel pain on 1473 professional (age range 18-43 years, mean $31.5 \pm 2.7$ ), semiprofessional and amateur players who came spontaneously to the University of Chieti for undifferentiated heel pain that limits their sports activity.

Patients were required to give written informed consent before participating in the study, in accordance with the Helsinki Declaration of the 41st World Medical Association General Assembly in Hong Kong in September 1989.

Soccer players of both sexes, with an age range of 18-43 years, were included in this study.

Exclusion criteria included absence of any pathological systemic condition (eg, rheumatoid arthritis) or pathological regional condition in the lower limbs (myofascial, neuropathic, orthopaedic) that might manifest with pain symptoms in the heel area, and absence of diabetes.

All patients have been visited by an expert orthopaedic specialist, and all diagnoses were made by clinical examination and diagnostic imaging.

Subjects were classified according to aetiological diagnosis; subjective pain was assessed using the Visual Analogue Scale (VAS) and disability using the Foot and Ankle Disability Index (FADI).

All data are presented as mean and SD.

All evaluated subjects were also submitted for physical examination of the lumbar spine using the Oswestry Disability Index; in some of those affected by heel pain, neurological examination revealed a history of sciatica.
When the pain originates in the low back and radiates down the leg and into the foot, this must be assessed for radiculopathy secondary to proximal nerve root pathology.

All subjects have been treated specifically according to the main diagnosis and reassessed at the end of treatment (T1) and after 6 months (T2), in the period of 1992-2012 (figure 1).

The rehabilitative therapeutic approach consists of the following:

- sural nerve compression-30 days of therapy with ice 15 min a day for 15 days more; laser therapy (10 sessions) combined with transcutaneous electrical nerve stimulation (TENS) or frequency rhythmic electrical modulation system (FREMS) sessions)

- compression of the abductor digiti minimi-30 days of therapy with ice $15 \mathrm{~min}$ a day for 15 days more; laser therapy (10 sessions) combined with daily stretching for 30 days and focal mechanical vibration (12 sessions) of the abductor of the hallux, gastrocnemius and plantar fascia; TENS or FREMs (10 sessions)

- atrophy and inflammation of the fat pad-30 days of therapy with laser therapy (8 sessions) combined with ultrasound (US) diathermy (10 sessions)

- plantar fasciitis - 60 days of therapy with focused extracorporeal shockwave therapy (ESWT) at a frequency of 2 sessions a week for 4 weeks, combined with daily stretching, then diathermy therapy (5 sessions)

- stress injury of the heel spur-40 days of therapy with cryo-magneto therapy (24 sessions) and focused ESWT (2 sessions a week for 4 weeks)

- stress fracture of the heel bone - 45 days of therapy with cryo-magneto therapy (30 sessions) and focused ESWT (2 sessions a week for 4 weeks)

- heel spur-30 days of therapy with US therapy, 2 sessions in the first week, with focused ESWT (2 sessions a week for 3 weeks).

For all the considered diseases, we recommend the use of customised foot orthoses to optimise ground foot reaction.

\section{RESULTS}

Clinical and instrumental examinations revealed that 960 of 1473 athletes (860 men, 100 women; age range 19-42.5, mean age $30.2 \pm 5.3$; mean height $176 \pm 5.8 \mathrm{~cm}$; mean weight $75.9 \pm 7.8 \mathrm{~kg}$ ) had inferior heel pain as the predominant disorder (table 1).

At T0 for this group, the mean VAS pain level was $6.7 \pm 1.55$ and the mean FADI score was $95.5 \pm 4.4$.

The prehabilitative therapeutic approach will consist of the following:

For all groups we recommend the use of customised foot orthoses to optimise ground foot reaction.

At the end of the protocol, players come back to play: 
1473 subjects with heel pain evaluated in the Department of Physical Medicine and Rehabilitation of "G. D'Annunzio" University in Chieti

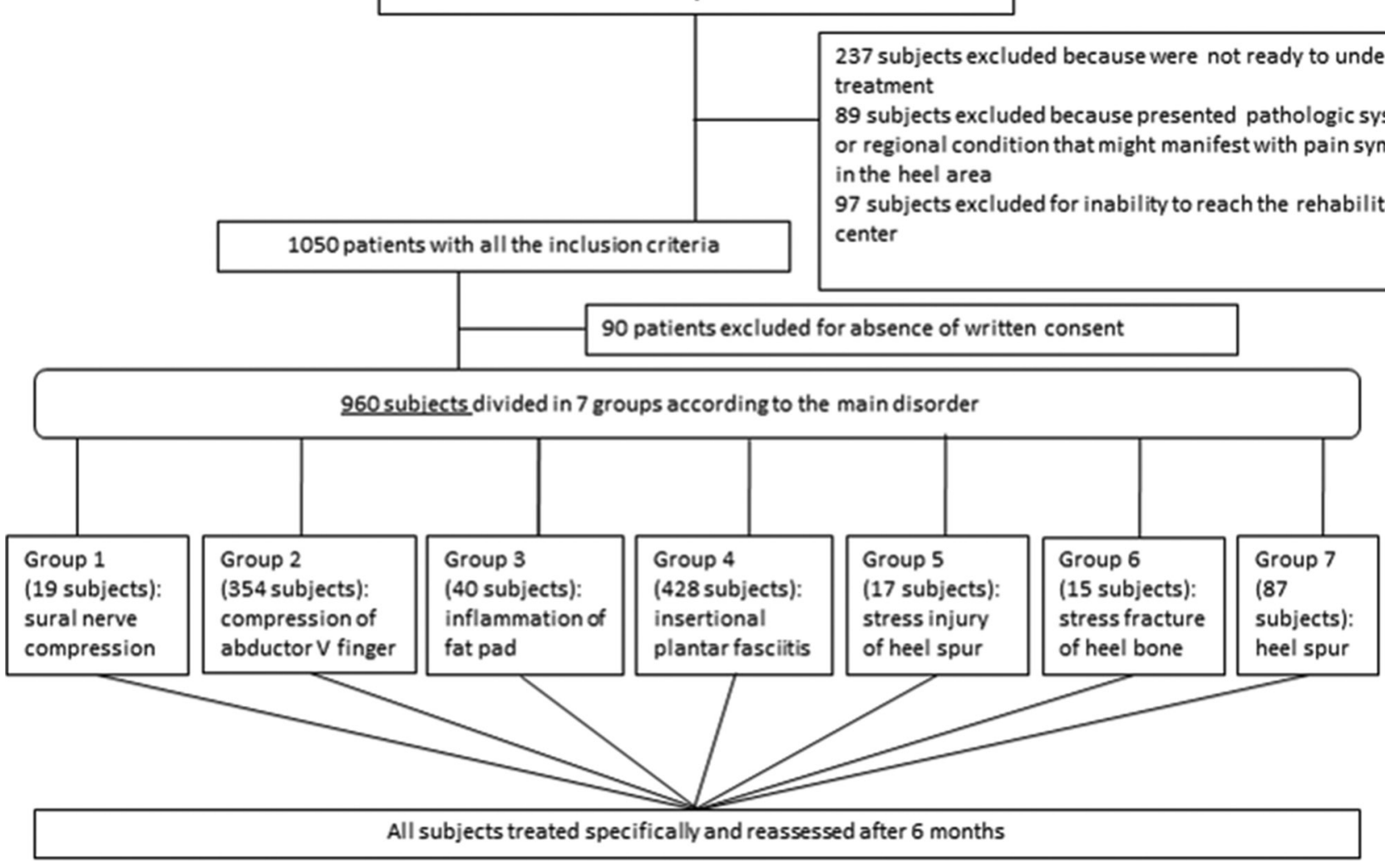

Figure 1 Flow chart of the study.

- sural nerve compression: return-to-play time $61 \pm 29$ days

- compression of abductor digiti minimi: return-toplay time $70 \pm 20$ days

- atrophy and inflammation of the fat pad: return-toplay time $53 \pm 37$ days

- plantar fasciitis: return-to-play time $58 \pm 32$ days

- stress injury of the heel spur: return-to-play time $64 \pm 26$ days

- stress fracture of the heel bone: return-to-play time $71 \pm 19$ days

heel spur: return-to-play time $62 \pm 28$ days.
On comeback to play, all the groups were re-evaluated with VAS to encode the persistence of pain (T1).

For each treatment group, statistically significant improvements in VAS were observed at T1 compared with T0 $(\mathrm{P}<0.05)$ (figures 2 and 3$)$.

The follow-up performed after 6 months from the end of the specific treatment showed persistence of a subjective pain of $1.8 \pm 0.45$ on VAS and a perceived disability of $122 \pm 4.9$ on FADI (tables 2 and 3 ).

For each treatment group, the VAS results obtained at T2 remained almost constant compared with $\mathrm{T} 0$, and we observed statistically significant improvements in FADI at

\begin{tabular}{lccccc}
\hline \multicolumn{7}{l}{ Table 1 } & Characteristics of patients according to pathology & & & \\
\hline Pathology & Patients $(\mathbf{n}), \mathbf{M}$ & Patients $(\mathbf{n}) \mathbf{~ W}$ & \multicolumn{1}{c}{ Age } & Height & Weight \\
\hline SNC & 15 & 14 & $34 \pm 5.9$ & $177 \pm 8.1$ & $79.5 \pm 7.7$ \\
\hline AbC & 322 & 32 & $30.7 \pm 5.5$ & $176.4 \pm 8.9$ & $76.1 \pm 8.7$ \\
AtFP & 40 & 0 & $32 \pm 3.4$ & $175.5 \pm 2.1$ & $77.2 \pm 2.3$ \\
PF & 367 & 61 & $29.7 \pm 4.7$ & $175.8 \pm 8.3$ & $75.1 \pm 7.6$ \\
\hline SIH & 17 & 0 & $33 \pm 5.2$ & $175 \pm 1.9$ & $77.2 \pm 2.5$ \\
SFH & 15 & 0 & $31.5 \pm 3.1$ & $175.3 \pm 3.2$ & $75.4 \pm 2.2$ \\
HS & 84 & 3 & $28.6 \pm 2.9$ & $175.4 \pm 7.9$ & $77.2 \pm 7.1$ \\
\hline
\end{tabular}

$\mathrm{AbC}$, abductor digiti minimi compression; AtFP, atrophy and inflammation of the fat pad; HS, heel spur; M, men; PF, plantar fasciitis; SIH, stress injury of the heel spur; SFH, stress fracture of the heel bone; SNC, sural nerve compression; W, women. 


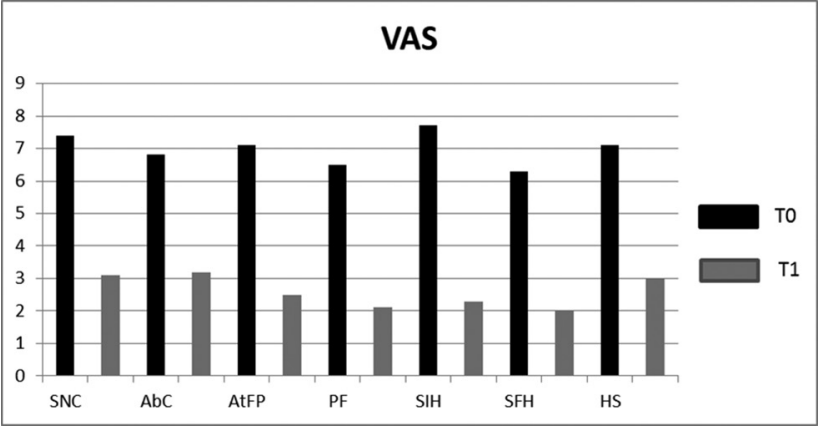

Figure 2 Trend of Visual Analogue Scale (VAS) scores of the seven groups of patients at T0 and T1. (From left to right: $\mathrm{SNC}$, sural nerve compression; AbC, abductor digiti minimi compression; AtFP, atrophy and inflammation of the fat pad; $\mathrm{PF}$, plantar fasciitis; SIH, stress injury of the heel spur; SFH, stress fracture of the heel bone; HS, heel spur\r\n).

T2 after 6 months compared with T0 $(\mathrm{P}<0.05)$ (figures 4, 5,6 and 7).

\section{DISCUSSION}

In the review of the literature, data show that heel pain is one of the most frequent reasons for medical examinations among players.

The purpose of this study was to examine the epidemiological difference of heel pain among soccer players and to propose specific therapeutic guidelines.

From our data, it appears that the inferior heel pain among soccer players is due to the local biomechanical stress, but there are also other causes; in the case of heel pain, from compression of the sural nerve and of abductor digiti minimi, there is an associated neurological cause.

The main nerves affected in heel pain are the posterior tibial (in tarsal tunnel syndrome), ${ }^{9}$ the medial calcaneal (heel neuroma), ${ }^{10}$ the medial plantar and the lateral plantar including the first branch to abductor digiti minimi, ${ }^{11}$ and the sural (including lateral calcaneal) nerves.

Nerves reported more commonly in plantar heel pain of neurological origin are the first branch of the lateral

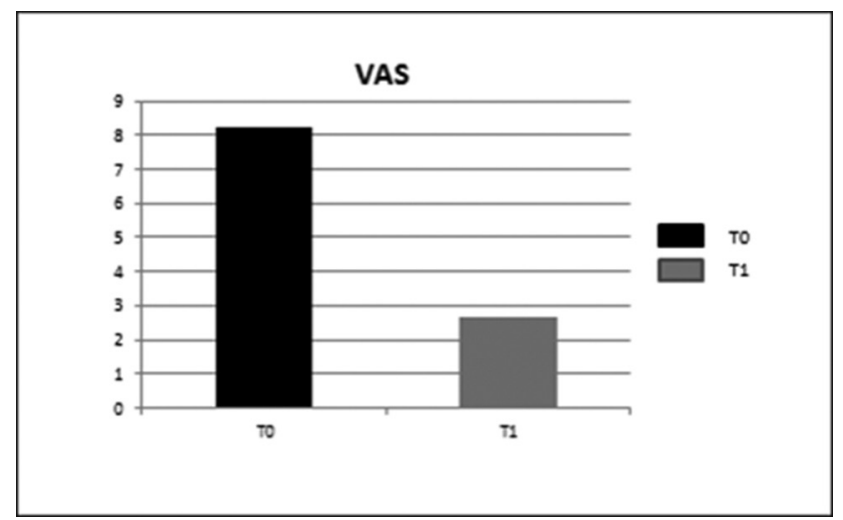

Figure 3 Mean Visual Analogue Scale (VAS) pain level of all groups at T0 and T1.
Table 2 VAS scores at T0 and T2 of the seven groups of patients

\begin{tabular}{llc}
\hline Pathology & VAS at T0 & VAS at T2 \\
\hline SNC & $7.4 \pm 1.1$ & $2.1 \pm 0.85$ \\
AbC & $6.8 \pm 1.3$ & $1.9 \pm 0.5$ \\
AtFP & $7.1 \pm 1.7$ & $2 \pm 0.55$ \\
PF & $6.5 \pm 1.1$ & $1.6 \pm 0.4$ \\
SIH & $7.7 \pm 1.8$ & $1.8 \pm 0.45$ \\
SFH & $6.3 \pm 1.2$ & $1.9 \pm 0.65$ \\
HS & $7.1 \pm 2.5$ & $1.7 \pm 0.45$ \\
\hline
\end{tabular}

AbC, abductor digiti minimi compression; AtFP, atrophy and inflammation of the fat pad; HS, heel spur; PF, plantar fasciitis; SFH, stress fracture of the heel bone; SIH, stress injury of the heel spur; SNC, sural nerve compression; VAS, Visual Analogue Scale.

plantar nerve (Baxter's nerve) ${ }^{12}$ and the medial calcaneal nerve.

Among the possible causal factors of nerve compression, as indicated in the literature among the possible causative factors of the nervous compression that deserve differential diagnosis, obesity, venous insufficiency, trauma and lesions that occupy space (as tumours), ${ }^{13}$ but in soccer players is linked to a history of mechanical sciatica.

Nevertheless the major studies have focused on the plantar fasciitis and possible treatments. Many studies have been on intrinsic and extrinsic risk factors related to the development of this disease, such as body weight and body mass index (BMI), the plantar load, the biomechanical overload factors, and the exercise mode. ${ }^{1415}$

Referring to the 2010 clinical practice guidelines developed by the CPG Heel Pain Committee of the ACFAS, ${ }^{6}$ the initial patient evaluation in suspected plantar heel pain consists of a significant history of isolated plantar heel pain, weightbearing pain after periods of rest, morning pain, barefoot walking or use of inappropriate shoes. If there are associated significant findings such as pain on palpation of the inferior heel or plantar fascia, obesity/

Table 3 FADI scores at T0 and T2 of the seven groups of patients

\begin{tabular}{lll}
\hline Pathology & FADI at T0 & FADI at T2 \\
\hline SNC & $99.3 \pm 4.3$ & $119 \pm 5.3$ \\
\hline AbC & $96.9 \pm 4.5$ & $122 \pm 4.7$ \\
AtFP & $97.8 \pm 4.5$ & $123 \pm 3.8$ \\
PF & $96.8 \pm 4.2$ & $122 \pm 4.0$ \\
SIH & $98.5 \pm 2.7$ & $122 \pm 4.4$ \\
SFH & $94.2 \pm 3.4$ & $123 \pm 4.3$ \\
HS & $97.8 \pm 3.2$ & $124 \pm 4.8$ \\
\hline
\end{tabular}

AbC, abductor digiti minimi compression; AtFP, atrophy and inflammation of the fat pad; FADI, Foot and Ankle Disability Index; $\mathrm{HS}$, heel spur; PF, plantar fasciitis; SIH, stress injury of the heel spur; SFH, stress fracture of the heel bone; SNC, sural nerve compression. 


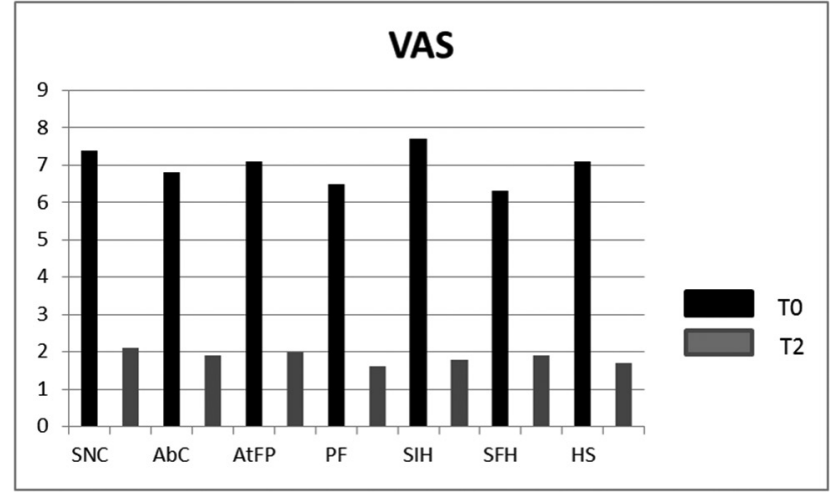

Figure 4 Trend of Visual Analogue Scale (VAS) score of the seven groups of patients at T0 and T2. (From left to right: $\mathrm{SNC}$, sural nerve compression; AbC, abductor digiti minimi compression; AtFP, atrophy and inflammation of the fat pad; $\mathrm{PF}$, plantar fasciitis; SIH, stress injury of the heel spur; SFH, stress fracture of the heel bone; HS heel spur).

high BMI, pronated foot architecture, localised swelling or atrophy of infracalcaneal fat pad, radiographs may be helpful. The treatment options suggested by evidencebased medicine as regards plantar heel pain have been graded according to three steps (figure 8).

The 2014 guidelines show a model that guides clinical decisions on the evaluation, diagnosis and treatment planning for individuals with heel pain/plantar fasciitis: in the diagnosis, the ICD category of plantar fasciitis and the associated International Classification of Functioning, Disability and Health impairment-based category of heel pain should be used. Clinicians should assess for diagnostic classifications other than heel pain/plantar fasciitis, including spondyloarthritis, fat pad atrophy and proximal plantar fibroma, when the individual's reported activity limitations or impairments of body function and structure are not consistent with those presented in the diagnosis/classification section of this guideline, or when the individual's symptoms do not resolve with interventions aimed at normalisation of body function. ${ }^{8}$

As regards therapy, Karl B Landorf, ${ }^{7}$ in a review of literature, reported the following: customised foot orthoses are more effective than sham orthoses in improving

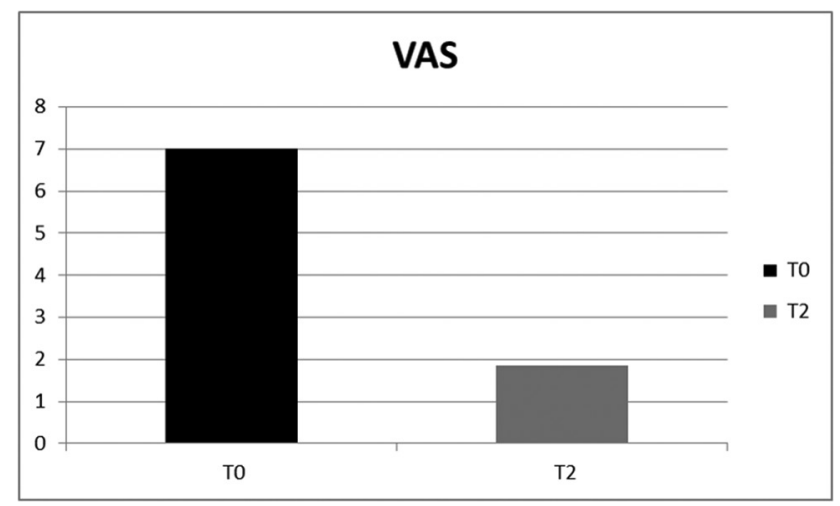

Figure 5 Mean Visual Analogue Scale (VAS) score of all groups at T0 and T2.

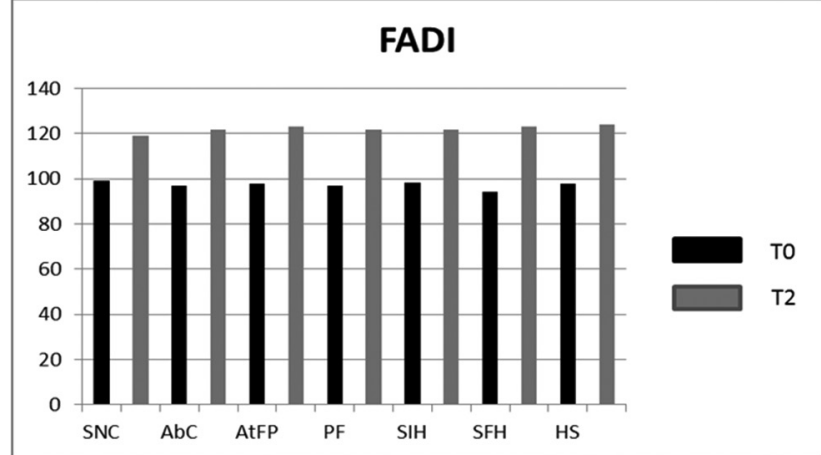

Figure 6 Trend of Foot and Ankle Disability Index (FADI) score of the seven groups of patients at T0 and T2. (From left to right: SNC, sural nerve compression; AbC, abductor digiti minimi compression; AtFP, atrophy and inflammation of the fat pad; PF, plantar fasciitis; SIH, stress injury of the heel spur; SFH, stress fracture of the heel bone; HS, heel spur).

functions up to 12 months in people with plantar heel pain, but with less effect in reducing pain; heel pads and heel cups are effective but lack randomised studies; taping may be more effective than no taping or sham taping in reducing pain in the short term (at 1 week); stretching exercises are more effective than no treatment or taping in reducing pain or improving function in people, but the evidence is weak and inconsistent; EWST may be more effective than placebo in reducing pain at 12 weeks in people with chronic heel pain, but there is a need for more randomised controlled trials; and corticosteroid injections may be associated with a high rate of plantar fascia rupture and other complications, which may lead to chronic disability in some people.

The authors of this study fully agree with the scientific evidence on the importance of therapeutic exercise, foot orthoses, and manual therapy and physical energies (eg, low-level laser therapy) in the treatment of heel pain/ plantar fasciitis, and that combined techniques have synergistic effects; it is necessary to emphasise that the weak evidence on the use of advanced physical energies might result in the initial definition of heel pain that excludes or considers in their own right the pain condition from nerve compression syndromes, inflammation

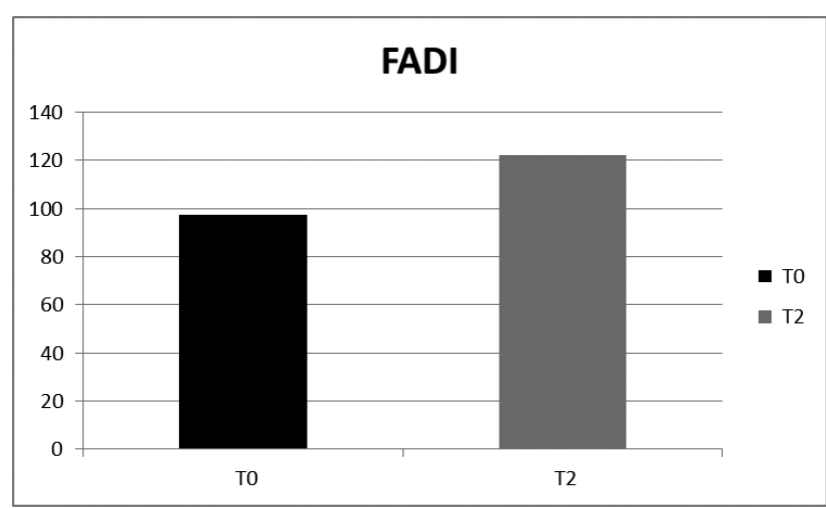

Figure 7 Mean Foot and Ankle Disability Index (FADI) pain level of all groups at T0 and T2. 


\section{Treatment of plantar heel pain}

\begin{tabular}{|l|}
\multicolumn{1}{|c|}{ First-line strategies } \\
- $\quad$ padding and strapping of the foot \\
stretching exercises of the plantar \\
fascia and the Achilles tendon \\
- $\quad$ recommended footwear \\
- $\quad$ oral anti-inflammatory drugs \\
- $\quad$ home physical therapy \\
corticosteroid injection in the area \\
of maximum tenderness
\end{tabular}

Addiction of:

- orthotic devices

- night splint to mantain the plantar fascia and the gastro-soleus complex in extension during sleep

- further corticosteroid injection

- injection of botulinum toxin

- cicle of physical therapy

- casting for 4 to 6 weeks

- use of specific footwear to immobilize or unload the foot during activity

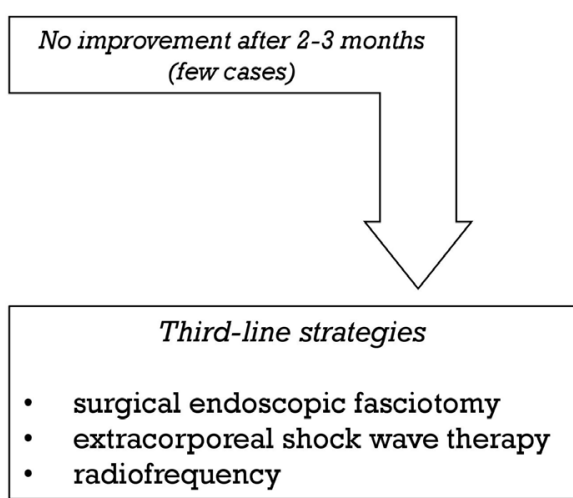

Figure 8 Treatment of plantar heel pain in three steps according to the 2010 CPG Heel Pain Committee of the American College of Foot and Ankle Surgeons guidelines. ESWT, extracorporeal shockwave therapy.

and atrophy of the fat pad, stress injury of the heel spur, stress fracture, and heel spur.

According to what was observed in the present study on the treatment of inferior heel pain, and considering the technological progress achieved in recent years in rehabilitation and biomedicine, we can now propose an integrated therapeutic approach to treatment of heel pain, properly differentiated according to the specific disorder, in order to improve the functional outcome of athletes, reducing and possibly removing the residual subjective pain, respecting the need to resume to sport as soon as possible and with minimal use of pharmacological treatments.

The proposed rehabilitative therapeutic approach, based on current knowledge and on progress of physical therapy, will consist of the following:

- Sural nerve compression-30 days of therapy with ice $15 \mathrm{~min}$ a day for 15 days more; laser therapy (10 sessions) combined with 'short voltage pulses controlled by software' with So.Co.Short electrotherapy (10 sessions); electro-neurofeedback in the last 10 days, concurrent with the return to sports. To realise an ideal return to sport, it is necessary to completely rest during the first 10 days, and restart training on day 11 and return to competitive activity on the 20th day.

- Compression of abductor digiti minimi-30 days of therapy with ice $15 \mathrm{~min}$ a day for 15 days more; laser therapy (10 sessions) combined with daily stretching for 30 days and focal mechanical vibration (12 sessions) of the abductor of the hallux, gastrocnemius and plantar fascia; 'short voltage pulses controlled by software' with So.Co.Short electrotherapy (10 sessions). To realise an ideal return to sport, it is necessary to completely rest during the first 10 days, restart training on day 11 and return to competitive activity on the 20th day.

- Atrophy and inflammation of the fat pad-30 days of therapy with laser therapy (8 sessions) combined with US diathermy (10 sessions). To realise an ideal return to sport, it is necessary to completely rest in the first 10 days, restart training on day 11 and return to competitive activity on the 20th day.

- Plantar fasciitis - 60 days of therapy with focused ESWT at a frequency of 2 sessions a week for 4 weeks combined with daily stretching, then Ultrasound Imperium (5 sessions). The subject may not even stop training and return to competitive activity after 10 days.

- Stress injury of the heel spur-40 days of therapy with cryo-magneto therapy (24 sessions) and focused ESWT ( 2 sessions a week for 4 weeks). It is advisable to restart training on day 21 and return to competitive activity on the 28th day.

- Stress fracture of the heel bone-45 days of therapy with cryo-magneto therapy (30 sessions) and focused ESWT ( 2 sessions a week for 4 weeks). To realise an ideal return to sport, it is necessary to completely rest with no load for the first 2 weeks, start walking from the third week and return to 
training after radiological evidence shows healing of fracture.

- Heel spur-30 days of therapy with US, 2 sessions in the first week, with focused ESWT ( 2 sessions a week for 3 weeks). The subject may return to competitive activity after the first 7 days of treatment.

For all the considered diseases, we recommend the use of customised foot orthoses in order to optimise ground foot reaction.

Contributors RS participated in the planning and coordination of the study. MM participated in its planning. SMC participated in the clinical part of the study, in particular on the medical aspect, and helped to write the manuscript and the statistical analysis. SMC participated in the clinical part of the study and in writing the manuscript. EA participated in the clinical part of the study. CR participated in the data collection. RGB participated in the clinical part of the study, in particular on the medical aspect, and in the coordination of the study. All authors read and approved the final manuscript.

Funding This research received no specific grant from any funding agency in the public, commercial or not-for-profit sectors.

Competing interests None declared.

Patient consent Obtained.

Provenance and peer review Not commissioned; externally peer reviewed.

Open Access This is an Open Access article distributed in accordance with the Creative Commons Attribution Non Commercial (CC BY-NC 4.0) license, which permits others to distribute, remix, adapt, build upon this work non-commercially, and license their derivative works on different terms, provided the original work is properly cited and the use is non-commercial. See: http://creativecommons.org/ licenses/by-nc/4.0/

C Article author(s) (or their employer(s) unless otherwise stated in the text of the article) 2018. All rights reserved. No commercial use is permitted unless otherwise expressly granted.

\section{REFERENCES}

1. Junge A, Cheung K, Edwards T, et al. Injuries in youth amateur soccer and rugby players - comparison of incidence and characteristics. Br J Sports Med 2004;38:168-72.

2. Suzue N, Iwame T, Kato $\mathrm{K}$, et al. Plantar fascia rupture in a professional soccer player. J Med Invest 2014;61:413-6.

3. Wong P, Hong Y. Soccer injury in the lower extremities. Br J Sports Med 2005;39:473-82.

4. Lareau CR, Sawyer GA, Wang JH, et al. Plantar and medial heel pain: diagnosis and management. J Am Acad Orthop Surg 2014;22:372-452.

5. FH F, David A, Stone MD. Sports injuries: mechanisms, prevention, treatment. 2nd edn. Pennsylvania, USA: Lippincott, Williams \& Wilkins, 1994.

6. Thomas JL, Christensen JC, Kravitz SR, et al. The diagnosis and treatment of heel pain: a clinical practice guideline-revision 2010. $J$ Foot Ankle Surg 2010;49:S1-19.

7. Landorf KB. Plantar heel pain and plantar fasciitis. BMJ Clin Evid 2015;25:1111.

8. Martin RL, Davenport TE, Reischl SF, et al. Heel pain - plantar fasciitis: revision 2014 clinical practice guidelines linked to the international classification of functioning, disability and health from the orthopaedic section of the american physical therapy association. J Orthop Sports Phys Ther 2014;44:A1-23.

9. Lau JT, Stavrou P. Posterior tibial nerve - primary. Foot Ankle Clin 2004;9:271-85.

10. Beito SB, Krych SM, Harkless LB. Recalcitrant heel pain. Traumatic fibrosis versus heel neuroma. J Am Podiatr Med Assoc 1989;79:336-9.

11. Watson TS, Anderson RB, Davis WH, et al. Distal tarsal tunnel release with partial plantar fasciotomy for chronic heel pain: an outcome analysis. Foot Ankle Int 2002;23:530-7.

12. Pfeffer GB. Plantar heel pain. Instr Course Lect 2001;50:521-31.

13. Hendrix CL, Jolly GP, Garbalosa JC, et al. Entrapment neuropathy: the etiology of intractable chronic heel pain syndrome. J Foot Ankle Surg 1998;37:273-9.

14. Johnston MR. Nerve entrapment causing heel pain. Clin Podiatr Med Surg 1994;11:617-24.

15. Shikoff MD, Figura MA, Postar SE. A retrospective study of 195 patients with heel pain. J Am Podiatr Med Assoc 1986;76:71-5. 